\title{
Can characters be classified directly as digits vs letters or must they be identified first?*
}

\author{
RAYMOND S. NICKERSON $\dagger$ \\ Bolt Beranek and Newman Inc. \\ 50 Moulton Street, Cambridge, Massachusetts 02138
}

\begin{abstract}
Two experiments were addressed to the question of whether or not Ss can distinguish between letters and digits without identifying the characters. In Experiment ISs attempted to identify noisy characters; a character was said to be implicitly classified correctly if the identification response, whether correct or incorrect, was in the same category vis-a-vis the letter-digit distinction as the stimulus. Implicit classification of characters for which the identification response was incorrect was very little better than chance. The task in Experiment II was to classify the characters directly. The explicit classification performance in this case was poorer than the implicit classification performance of Experiment I. The results were taken as evidence that Ss could not distinguish between letters and digits unless they could identify the characters.
\end{abstract}

The fundamental nature of classification, or categorization, and its importance for perception and cognition are widely recognized. Bruner, Goodnow, and Austin (1956) have argued convincingly that, without the ability to place different stimuli in equivalence classes, one would not be able to cope with the infinite variety of sensory inputs with which one is continually confronted. The process by which human beings classify stimuli is still poorly understood, however, in spite of a long history of research and the impetus that this research has recently received because of the interest in developing pattern-classification procedures for machines.

There are two ways one might determine that a stimulus belongs to a specified class: (1) determine that it has a feature or set of features that defines class membership (a whale is a mammal because it is warm-blooded, has skin, nurses its young, etc.) or (2) determine that it is a member of a class that is known to be a subclass of the class in question (a Stetson is an article of clothing because it is a hat and a hat is an article of clothing). Any given stimulus might be classified in either way, and, of course, to say that the second procedure is used in any particular case begs the question of how membership in the subclass is itself determined.

In general, it would seem that the determination of membership in a higher order (more inclusive) class should be easier than the determination of membership in a lower order class, inasmuch as the more inclusive the class, the smaller the number of features that define membership. An entity that satisfies the criteria for membership in a subclass necessarily satisfies those for membership in the class to which the subclass itself

*This study was supported by the United States Air Force Office of Scientific Research under Contract F44620-69-C-0115. Barbara Freeman assisted in the collection of data.

$\dagger$ Requests for reprints should be sent to Raymond $S$. Nickerson, Bolt Beranek and Newman Inc., 50 Moulton Street, Cambridge, Massachusetts 02138 . belongs; whereas the converse is not true. It is easy to think of examples, however, for which it appears to be the case that people determine membership in a higher order class by first recognizing that the item belongs to a subclass of the class in question, that is to say, by the second of the ways mentioned above. One probably decides that an object is, say, a piece of fruit by recognizing it as, say, an orange and applying the knowledge that oranges are fruit.

The latter classification procedure would be appropriate when the class in question is not easily distinguished from another class except by listing its members. Cases in point might be the two classes of visual symbols, digits and letters. It seems likely that one recognizes a symbol as a member of the class of digits, as opposed to that of letters, by recognizing it as a member of one of those subclasses (e.g., the subclass of all 2s) that he knows to be included in the class of all digits. The plausibility of this conjecture rests on the assumption that a feature testing procedure that would suffice to distinguish reliably between digits and letters would probably suffice to identify the characters. (The term "identification" is used here to denote the more exclusive classification, but it should be borne in mind that this is a form of classification no less than the other.)

A test of the idea that the classification of characters as digits vs letters is based on identification of the characters was made by Dick (1971). He found that Ss could name visually presented characters from 100 to $200 \mathrm{msec}$ faster than they could report whether the characters were digits or letters and concluded in favor of the classification via identification hypothesis.

In direct opposition to this view, the results of several experiments suggest that alphanumeric characters may be classified vis-a-vis the digit-letter distinction in less time than is required to identify them by name. Brand (1971) and Ingling (1972) reported that Ss can search for a target character in a field of nontarget characters faster and more accurately when target and nontarget 
characters are from different categories (digits vs letters) than when they are from the same category. Both investigators concluded that, when nontarget characters differ in class from the target, they can be rejected on this basis; classification itself, they suggested, must be an extremely rapid process that does not depend on identification. Neither ventured a guess concerning how the classification is accomplished, but both dismissed the possibility of a distinctive feature analysis. To rule out this possibility, Ingling varied the similarity between target and nontarget items independently of category; for each of the conditions representing correspondence between target and nontarget categories, two subconditions were used, in one of which target and nontarget characters were physically similar and in the other of which they were physically dissimilar. The correspondence between target and nontarget items with respect to physical features did not significantly affect performance.

In further support of the idea that classification precedes, or occurs in the absence of, identification, Brand showed that some Ss (5 out of 12 in her experiment) can scan a field of one class of characters for a target of the other class as rapidly when the target is specified only with respect to class as when it is a specific character. A similar result has been reported by Sperling, Budiansky, Spivac, and Johnson (1971). When given the task of reporting the row and column location of a digit that occurred in one of a rapidly presented sequence of letter arrays, Ss did about as well when looking for any digit as when looking for a particular one. Sperling et al did not conclude that performance of their Ss was based on classification as opposed to identification; in fact, they preferred to assume that Ss analyzed target digits sufficiently completely to identify them even when this was not required by the task. They took the failure to obtain a difference between the oneand many-target conditions as evidence that target identification was based on a parallel search in which memory representations of all 10 digits are compared simultaneously with a character on the display. The claim here is not that a classification hypothesis is favored by the data of Sperling et al, but only that the assumption that Ss based their decisions on an analysis that was sufficient to distinguish digits from letters (and not necessarily sufficient to identify characters) in both the one- and many-target conditions is an alternative explanation of their results.

Posner (1970) has also concluded that Ss can distinguish between digits and letters without identifying the characters. The main basis for the conclusion in this case was the fact that it took no longer for Ss to decide that two simultaneously presented characters were in the same class (both letters) when they were drawn from a set that contained confusable items than when they were relatively easy to identify. Posner reasoned that, if one must always identify (name) a character before classifying it, the decision that two characters are in the same class should take longer the more difficult the characters are to identify.

Still another relevant finding is one obtained by Dick (1969), who discovered that class of character (digit vs letter) can be at least as effective an identifier of items to be recalled in a Sperling-type partial-report recall task as are location and color. Dick and Ingling saw different implications in this finding. Dick took it as evidence that selectivity is located at a relatively late stage of processing, He conceptualized the process in terms of Atkinson and Shiffrin's (1968) model as follows. The items are held momentarily in a sensory register from whence they are identified. A representation of the identity information is transferred to short-term storage, where the classification is accomplished after the transfer has been made. Character class can then be used to retrieve selectively from short-term storage in order to make the report. In particular, Dick assumed that items were classified after being identified: "The class-of-item group must first identify or name the item before classifying it [p. 283]." Ingling interpreted the finding as favoring the view that selectivity occurred at an earlier stage of processing. In particular, she suggested that the characters may be read out of iconic storage by symbolic category and that this step not only precedes but facilitates identification.

If characters can indeed be classified as digits or as letters before being identified, it is of interest to ask how the classification is accomplished. The question is particularly intriguing if, as Ingling suggests, the classification is not based on an analysis of features that would be sufficient to differentiate one class from the other. It would seem reasonable to expect that, whatever the nature of the classification process, it should require less stimulus information than does identification. For if classification required as thorough an analysis of a character as does identification, we would not expect it to take less time. The experiments reported here were addressed to the question of whether characters can be classified vis-a-vis digit vs letter categories on the basis of less, or more noisy, information than is required to identify them.

It must be emphasized that this study focuses on the digit vs letter distinction. The task of distinguishing digits or letters from nonalphanumeric shapes may involve quite different processes than those involved in distinguishing digits and letters from each other. What makes the latter task particularly interesting is the possibility that these two categories are not readily distinguishable by simple feature testing.

\section{EXPERIMENT 1}

The purpose of the first experiment was to obtain some evidence on the question of the extent to which digits and letters may be considered to be distinctively different visual patterns. Do they differ in discriminability? Are characters of one class more 
readily confused with members of the same class than with members of the other class? How well does the confusability between pairs of characters correlate with a crude but objective measure of similarity, and does the answer to this question depend on whether the characters are from the same or from different classes?

\section{Method}

On each trial, $\mathrm{S}$ was shown a "noisy" digit or letter comprised from a subset of the dots of a 7 by 5 matrix. The character was displayed on a computer-controlled cathode ray tube; the matrix measured $11 \times 8 \mathrm{~mm}$ and was viewed from a distance of about $1 \mathrm{~m}$. The task was to identify the digit or letter by name. The display on any given trial represented the set sum, or union, of a set of dots defining a character and a set defining a noise pattern. A new noise pattern was generated for each trial, the locations of the noise dots being chosen at random, independently of the choice of character. Examples of noiseless and noisy characters are shown in Fig. 1.

Three levels of noise, 8,14 , and 20 dots, were used in order to insure a relatively wide range of stimulus discriminability. These levels were chosen on the basis of some pilot data that suggested that they would produce performance measures on the order of $75 \%, 50 \%$, and $25 \%$ correct. As it turned out, the obtained means for the 8-, 14-, and 20-dot noise levels were $75 \%, 49 \%$, and $28 \%$, respectively.

On each trial a character plus noise pattern was displayed for $1 \mathrm{sec}$. The $\mathrm{S}$ indicated the character that he thought he saw by typing it on a teletypewriter. He further indicated that he was satisfied with his choice by typing a carriage return. He was allowed as much time as he wished to make his choice and was permitted to change it as often as he liked before striking the carriage return. Once the carriage return had been struck, the last character that had been typed was considered to be the answer. One and one-half seconds before the onset of the character, the display area was marked by the illumination of four dots positioned at the corners of the 9 by 7 matrix that would have been formed by expanding the 7 by 5 matrix of the display area one row or column in every direction. The marker dots remained on for $1 \mathrm{sec}$ and there was a .5-sec delay between their offset and the onset of the character. The time between S's response (carriage return) and the onset of the four marker dots for the following trial was constant at $1 \mathrm{sec}$.

Each of 12 Ss had six experimental runs, two with each level of the noise variable. The experiment was counterbalanced with respect to the order in which Ss encountered the various noise levels. Every character (10 digits and 26 letters) occurred twice within a single experimental run. The intensity of the display was set at a comfortable level and the experiment was run in a normally lighted room. Before the first experimental session, $S$ was given a familiarization run in which he was shown each character of the set, without noise, in turn.

\section{Results}

Because every character occurred twice in each run, about $28 \%$ of the stimuli were digits and $72 \%$ were letters. About $29 \%$ of the responses were digits and $71 \%$ letters. The largest deviation from this split was obtained with the 20 -dot noise level, in which case $32 \%$ of the responses were digits and $68 \%$ letters.

The probability that a character would be identified correctly, given that it was a digit, was $.72, .48$, and .30 for the 8-, 14-, and 20-dot noise levels, respectively. The corresponding figures for letters were $.76, .50$, and .27 . In short, digits and letters did not differ greatly in terms

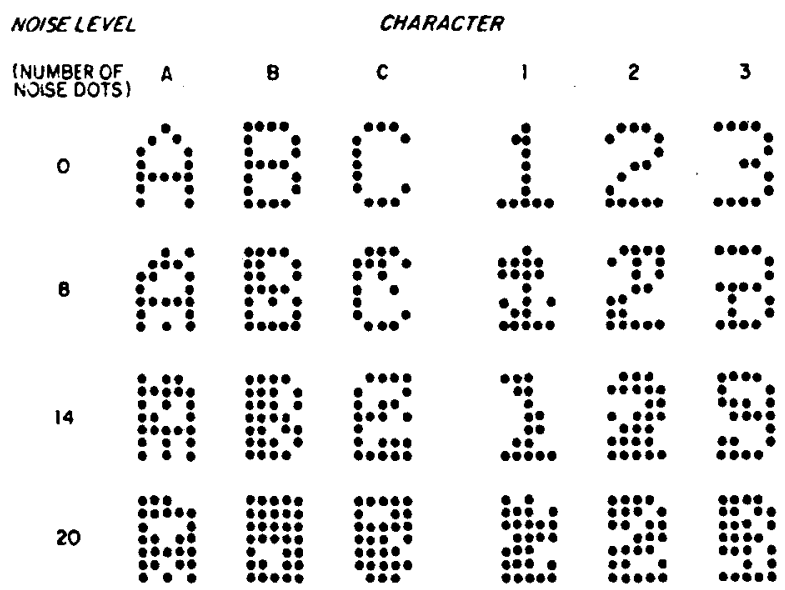

Fig. 1. Examples of the characters used in this experiment and the effects of the different noise levels.

of the accuracy with which they were identified.

The probability that an erroneous response would be in the same class as the stimulus was $.59, .62$, and .61 for the 8-, 14-, and 20-dot noise levels, respectively. In order to evaluate the significance of these figures, it is necessary to take into account the fact that letters occurred more frequently than did digits, both as stimuli and as responses on trials for which the response was incorrect (as well as on trials for which the response was correct). Considering only those trials on which incorrect responses occurred, the percentages of stimuli (responses) that were digits were 31 (29), 29 (30), and 27 (32) for the 8-, 14-, and 20-dot noise levels, respectively. Given these frequencies of occurrence of the stimuli and responses of the two types, the hypothesis of independence between stimulus and response classes would predict that the probabilities of stimulus and response being in the same class would be $.58, .59$, and .58 for the $8-, 14-$, and 20-dot noise levels, respectively. The differences between these values and those obtained are statistically significant for the 14-dot $\left(\chi^{2}=6.08, \quad p<.025\right)$ and the 20-dot $\left(\chi^{2}=4.85\right.$, $p<.05)$ noise levels. More important than the statistical significance of the differences, however, is the fact of their small size. If characters are more likely to be confused with other characters of the same class than with characters of the opposite class, these results suggest that the effect is an extremely small one.

The method that was used to construct the stimulus characters in this study permitted the development of a simple, if crude, objective measure of the degree of similarity between two characters. The measure was defined as

$$
S(X, Y)=\frac{n_{x, y}}{n_{x, y}+n_{x, \bar{y}}+n_{\bar{x}, y}}
$$

where $n_{x, y}=$ the number of dots that $x$ and $y$ have in common, $\mathrm{n}_{x, \bar{y}}=$ the number of dots that $\mathrm{x}$ has and $\mathrm{y}$ does not, and $n_{\bar{x}, y}=$ the number of dots that $y$ has and 
Table 1

Three Indexes of Similarity for Digits and Letters (See Text for Explanation)

\begin{tabular}{llll}
\hline & $\mathrm{S}_{\mathrm{S}}$ & $\mathrm{S}_{\mathrm{D}}$ & $\mathrm{S}_{\mathrm{T}}$ \\
\hline Digits & .31 & $.32^{*}$ & .32 \\
Letters & .37 & $.32^{*}$ & .36 \\
All Characters & $.35 \dagger$ & .32 & .34 \\
\hline
\end{tabular}

"These two numbers have to be the same; they both represent the mean similarity between all possible digit-letter pairs. tThis number is the weighted mean of the two numbers above it, each of the within-class means being weighted by the number of comparisons involved.

$x$ does not. The virtue of this index is its simplicity and lack of ambiguity. No claim is made for its psychological validity relative to that of other indices that might be developed on the basis of commonality of features, such as horizontal or vertical line segments, angles, arcs, etc. However, the fact that all characters were presented in exactly the same location and orientation gives some credence to the index as a meaningful measure to apply in this situation.

Note that the index may range from 0 to 1 , with 0 representing the case in which two patterns have no dots in common and 1 the case in which the two patterns are identical. In fact, the index ranges from .03 (I-N) to .89 (D-O). All of the following pairs have indexes less than .10: H-I (.04), O-Y (.04), N-W (.04), C-Y (.05), 1-4 (.08), A-T (.08). The following pairs have indexes of at least .80: B-D (.81), C-O (.31), F-P (.81), P-R (.83), 8-B (.85), 8 -S (.88).

In order to get an indication of the extent to which the similarity index could be used as a predictor of confusability between specific pairs of characters, pairs having the 10 lowest similarity indexes were compared with those having the 10 highest indexes with respect to the frequency with which the items of a pair were confused. (Correlation coefficients computed on all possible pairs would have been misleading, because of the very large incidence of pairs that were confused only once or not at all.) For the 10 pairs with the lowest similarity indexes, the mean numbers of confusions (of a possible 48) were $1.3,1.5$, and 1.2 for the 8-, 14-, and 20-dot noise levels, respectively. Over half of all the confusions that occurred between items of these pairs are accounted for by the single pair N-W, an apparent example of a case for which the similarity index is not a good predictor of confusability. Exclusion of this pair reduces the means to $.3, .6$, and .8 . The corresponding figures for the pairs having the 10 highest similarity indexes are $6.5,10.3$, and 8.2. Thus, the index appears to be a reasonably good predictor of confusability, at least in the aggregate.

The fact that the mean number of confusions between the highly similar pairs did not increase monotonically with noise level may seem to be incongruous with the fact that the frequency of incorrect responses did increase monotonically with this variable. An examination of the confusion matrices suggested one plausible explanation of this result. It appears that any increase in noise level increases the probability of an error and that, as long as the level is a moderate one, the increasingly frequent erroneous responses tend to be those that can be accounted for by the similarity between the stimulus and response characters. As the noise level is further increased, however, the pattern more frequently is sufficiently obscured to preclude recognizing the stimulus even as one of a small set of possibilities and, consequently, the errors become more and more random.

Increases in noise level did not drastically change the relative identifiability of the individual characters; while the identifiability of every character was decreased by increasing the noise level, characters that were among the more difficult to identify at one noise level tended also to be among the more difficult at another. This assertion is based on correlations between the number of correct identifications of each character for every pairwise comparison of noise levels. The Pearson is for the $8.14,14.20$, and 8.20 pairings were, respectively, $.85, .86$, and .73 .

Several computations involving the similarity index were made. Three mean indexes were computed for every character: $S_{S}, S_{D}$, and $S_{T}$. $S_{S}$, for a given character, is the mean pairwise similarity between the character and all other characters of the same class. That is, $S_{S}$, for a given character, is the mean of all the similarity indexes involving that character and every other character within the same class. $S_{D}$ and $S_{T}$ are similarly defined, except that $S_{D}$ represents the mean pairwise similarity between a character and all characters of the other class and $S_{T}$ is computed using all characters from both classes. Table 1 gives the mean values of $S_{S}, S_{D}$, and $S_{T}$ for digits, letters, and all characters combined. The following observations may be made from this table concerning aggregate interitem similarities of alphanumeric characters, inasmuch as these are reflected by the index defined above. (a) Digits are not more similar to digits than to letters. (b) Letters are somewhat, though not much, more similar to letters than to digits. (c) In general, characters are only slightly more similar to characters of the same class than to characters of the other class $\left(S_{S}\right.$, all characters, vs $S_{D}$, all characters). (d) Digits have a slightly lower aggregate similarity index than do letters $\left(\mathrm{S}_{\mathrm{T}}\right.$, digits, vs $\mathrm{S}_{\mathrm{T}}$, letters). (e) All of the differences in the table are small, suggesting that the assumption that digits and letters belong to distinctively different classes of visual patterns is probably not valid.

$\mathrm{S}_{\mathrm{T}}$ for a given character may be taken as a gross measure of the similarity of that character to all other alphanumeric characters. In order to determine whether this measure would be a good predictor of confusability, it was correlated with the number of correct identifications for each character pooled over the three noise levels. The Pearson $r$ was -.23 . The correlation is 
in the expected direction: however, it is not significantly different from 0 and, in any case, is too small to justify the claim that one of these variables is a good predictor of the other. A plausible explanation of this is the fact that the composite measure $\left(\mathrm{S}_{\mathrm{T}}\right)$ is relatively insensitive to individual pairwise similarities that may be unusually high. For example, $\mathrm{F}$ does not have a particularly high $\mathrm{S}_{\mathrm{T}}(.38)$, which is to say that, on the average, it is not highly similar to many other characters but it is very similar to the particular character P; and the latter fact is apparently at least as important a determinant of the identifiability of $F$ as is the former.

In an experiment addressed to some issues of visual encoding, Snodgrass (1972) divided digits into three classes on the basis of whether they were composed of straight lines $(1,4,7)$, curves $(3,6,8,9)$, or both $(2,5)$. Pairwise similarity was considered to be greatest when the members of the pair were selected from within the same class (e.g., 1, 4 or 3,6) and least when one item of the pair was selected from the set composed of straight lines and the other from the set composed of curves (e.g., 1, 3 or 6,4). When a member of either the straight set or the curved set was paired with a 2 or a 5 , the degree of similarity was considered to be intermediate. Snodgrass found that the time required to decide that two digits differed varied directly with similarity thus defined, although the effect was small. In terms of the index defined above, the mean pairwise similarity for the most, intermediate, and least similar pairs as defined by Snodgrass is $.363, .362$, and .220 , respectively. Thus, the index does not discriminate between the highest and intermediate degrees of similarity, but it does discriminate between both of these and the lowest. The .363 measure for the within-class pairs is as low as it is because of the fact that the similarity index is very small (.105) for pairs drawn from within the straight-line class; the figure for pairs drawn from within the curved-line class is 480 . In this experiment, the average number of confusions (of a possible 48) between members of most, intermediate, and least similar pairs as defined by Snodgrass was 6,7 , and 4 , respectively. Thus, again it appears that Snodgrass' rule discriminates between highest or intermediate and lowest degrees of similarity more effectively than between the highest and intermediate degrees.

\section{EXPERIMENT II}

In Experiment I, the task was to attempt to identify noisy characters by name. Performance was evaluated not only in terms of success on the identification task but also in terms of the accuracy of the implicit classification of characters vis-a-vis the distinction between digits and letters. (We will say that a character was implicitly classified correctly if S's identification response, whether or not correct, was in the same category as the stimulus.) It was found that the implicit classification of stimuli for which the identification response was incorrect was very little better than chance. This would seem to rule out the possibility that Ss classify characters as digits or letters as a first step in the identification process in order to reduce the number of alternatives from which the identification response must be selected (Ingling, 1972). Or at least, if they did follow this procedure, it was not very effective in this case.

It is tempting to go one step further and conclude that, if a character cannot be identified, it cannot be correctly classified. That conclusion is probably not warranted, however, on the basis of the results of Experiment I. The Ss were not asked to classify the characters explicitly, and the assumption that had they been asked to do so their choices would have invariably been those implicated by their identification responses is not compelling. In fact, the assumption begs the question of the relationship between identification and classification and rests on the view that these processes are links in a serial chain. (The assumed order of the processes is irrelevant here.) If classification and identification were independent processes, possibly occurring in parallel, we might expect that the confusions that would be made in an identification task would be strictly a function of physical similarity among characters. In this case, what the results of Experiment I would establish is that the digits and letters that were used in this study are not perceptually distinct classes of patterns. The purpose of Experiment II was to provide some data that, together with those obtained in Experiment I, could be used to test the validity of the conjecture that, if a character cannot be identified, it is unlikely that it can be correctly classified in terms of the digit-letter distinction.

\section{Method}

Experiment II was identical in all respects to Experiment I, except that the task was to attempt to classify each character as a digit or a letter rather than to identify it, and only $6 \mathrm{Ss}$ were run instead of 12. Each $\mathrm{S}$ had a familiarization run, followed by six experimental runs, two with each noise level.

\section{Results}

Figure 2 compares the explicit classification performance of Experiment II with the implicit classification performance of Experiment I. T tests verified that the difference in favor of Experiment $I$ is statistically significant $(p<.01)$ at every noise level. This result was unexpected, and some consideration was given to the possibility that it was an artifact of $S$ sampling. In Experiment I, five of the Ss were high school students and all but one of the rest were laboratory personnel; the remaining $\mathrm{S}$ was the spouse of an employee. In Experiment II, all six of the Ss were high school students. If the performance of the high school students as a group differed from that of the laboratory personnel on these tasks, this could account for the differences represented in Fig. 2. To check this 


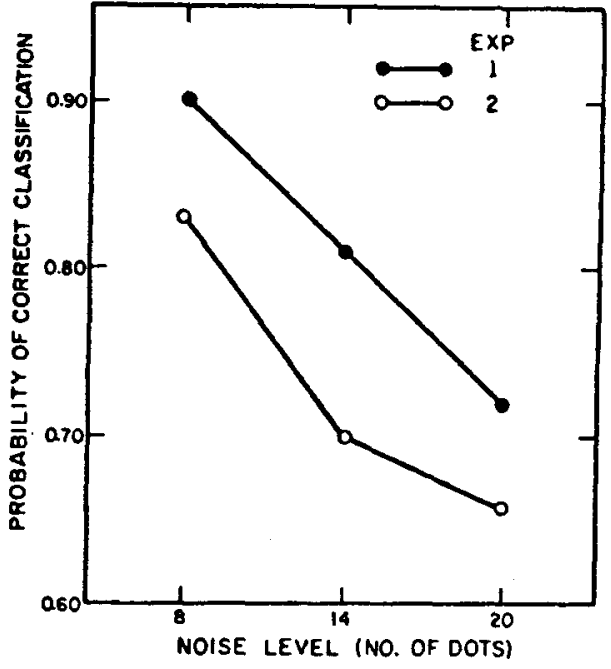

Fig. 2. The probability of a correct implicit (Experiment I) or explicit (Experiment II) character classification as a function of noise level.

possibility, the classification performance of the high school students in Experiment I was analyzed separately from that of the other Ss. The scores of the students were for two noise levels identical to those shown in Fig. 2, and in the third case differed by only one percentage point.

\section{DISCUSSION}

In Experiment $I$ it was found that the distribution of incorrect responses with respect to digit and letter categories was relatively independent of the distribution of stimulus characters with respect to these categories. That is to say, given that $\mathrm{S}$ failed to identify a character correctly, the probability that his erroneous response was at least in the correct category vis-a-vis the digit vs letter distinction was little better than chance. This is not consistent with the hypothesis that one classifies a character before identifying it, unless one makes the assumption that the probability of correct identification, given a correct classification, is very close to 1.0 . The result that was obtained is more in keeping with the hypothesis that Ss identify characters directly and, if the task requires that they classify them, that they classify them by identifying them first.

In Experiment II Ss were asked to classify the characters explicitly and identification was not required. The point was to determine whether their classification performance would be better than the implicit classification performance obtained in Experiment $I$, almost all of which was accounted for by correct identifications. Classification was not better in Experiment II than in Experiment I, suggesting again that Ss could not distinguish effectively between digits and letters unless they could identify the characters.

The fact that the classification performance was poorer in Experiment II than in Experiment I was not anticipated. The expectation was that classification performance would be either the same in both experiments (if characters can be classified only when they can be identified) or perhaps better in Experiment II (if characters can sometimes be classified even when they cannot be identified). The question now is, if the first hypothesis is correct, how is it that the classification performance in Experiment II, in which Ss were required to classify the characters directly without overtly identifying them, was not as good as the implicit classification performance of Experiment I?

The expectation for identical performance in both experiments follows from the assumption that in the explicit classification task $S$ invariable bases his choice of category on the character that he would have emitted as a response had he been asked to identify the stimulus. Perhaps this is not a reasonable assumption. Given the noisy characters that were used in these experiments, S's identification response undoubtedly would often be made on the basis of something less than $100 \%$ certainty in the correctness of his choice. Presumably, when his task is to identify a character, he selects the alternative that he considers to be the most probable, given the cues that are available to him. To opt for the most probable character in the classification task, however, may be a less reasonable strategy to follow. Suppose, for example, that $S$ feels that a particular character could be any one of a set of three specific characters, one of which is a letter and two of which are digits. Suppose further that he feels it is most likely to be the letter (and in fact would give the name of that letter as a response if he were required to identify the character instead of to classify it) but that the likelihoods for each of the two digits are only slightly smaller than that for the letter and that their union is larger. That is, whereas the letter is considered to be the most likely of the three candidates, the probability that the character is one of the two digits may be greater than the probability that it is that particular letter. Thus, $\mathrm{S}$ might reasonably decide in favor of the digit category, even though he would have responded with a letter had he been asked to identify the character. It does not follow from the assumption that this situation occurs, that classification performance should be poorer when it is explicit, as in Experiment II, than when it is implicit, as in Experiment I. In fact, one might argue that, on the contrary, classification performance should be better when $\mathrm{S}$ is permitted to weigh all the cues at his disposal in this fashion. The point is that the assumption that classification is based on identification does not necessarily imply that, given a specific stimulus, the category chosen in a classification task must be the category of the character that is chosen in an identification task.

A second possible explanation of the difference in classification performance between Experiments I and II is that the tasks may have been differentially motivating. If Ss perceived the classification task to be intrinsically 
simpler than the identification task, it may be that they attended to it less carefully and, as a consequence, did not carry the analysis of the individual characters to the same degree as in the identification task.

A third possibility has to do with guessing behavior. As was noted in the discussion of the results of Experiment I, what to expect in terms of proportion of correct classification responses assuming complete independence between stimulus and response categories depends on the proportions in which one's responses are distributed between the two response categories. Inasmuch as about $72 \%$ of the stimuli ( 26 out of 36 ) were letters in both of these experiments, the expected number of fortuitously correct classifications ranges from $28 \%$, if one were invariably to select "digit," to $72 \%$, if one always responded "letter." In the absence of any stimulus information, the more frequently one selects the letter response, the better he will do. Assuming that some portion of the responses in these experiments were pure guesses, any factor that would encourage $S$ to select the letter response in these cases would improve his performance-at least as measured by percent correct classifications. Table 2 shows the classification confusion matrices (in percentages) for both experiments and each noise level. The thing to notice is that response distributions differed somewhat between the two experiments. In Experiment I the proportions of responses falling in the two categories were very close to the proportions of stimuli in these categories. In Experiment II there was an increase in the relative frequency of digit responses. If one assumes that this difference is due to a different distribution of guesses, one can thereby account for the differences in classification scores. Moreover, this assumption can be made at least plausible. Inasmuch as Ss in Experiment I had to respond with the names of characters and they knew that all characters were equally represented, they may have been prompted to attempt to use the character names with roughly equal frequency - which would have the effect of making the distribution of responses over the digit and letter categories comparable to that of the stimuli. It seems less likely that in Experiment II Ss would have been prompted to maintain a 10 to 26 ratio of digit to letter responses, and a shift of this ratio in the direction of something slightly closer to 50-50 perhaps should not be surprising.

In any case, whatever the explanation of the fact that classification performance was poorer in Experiment II than in Experiment $I$, the important point is the fact that it was not better. The results of the two experiments in combination lead to the following conclusions: (1) Alphanumeric characters are not appreciably more confusable with characters from the same class than with characters from the other class, and (2) it is not possible to classify characters vis-a-vis the digit-letter distinction on the basis of less stimulus information than is required to identify the characters.

It is possible that these results are attributable in part
Table 2

Classification Confusion Matrices (in Percentages) for Both Experiments and Each Noise Level

Each group of scores is organized as follows:

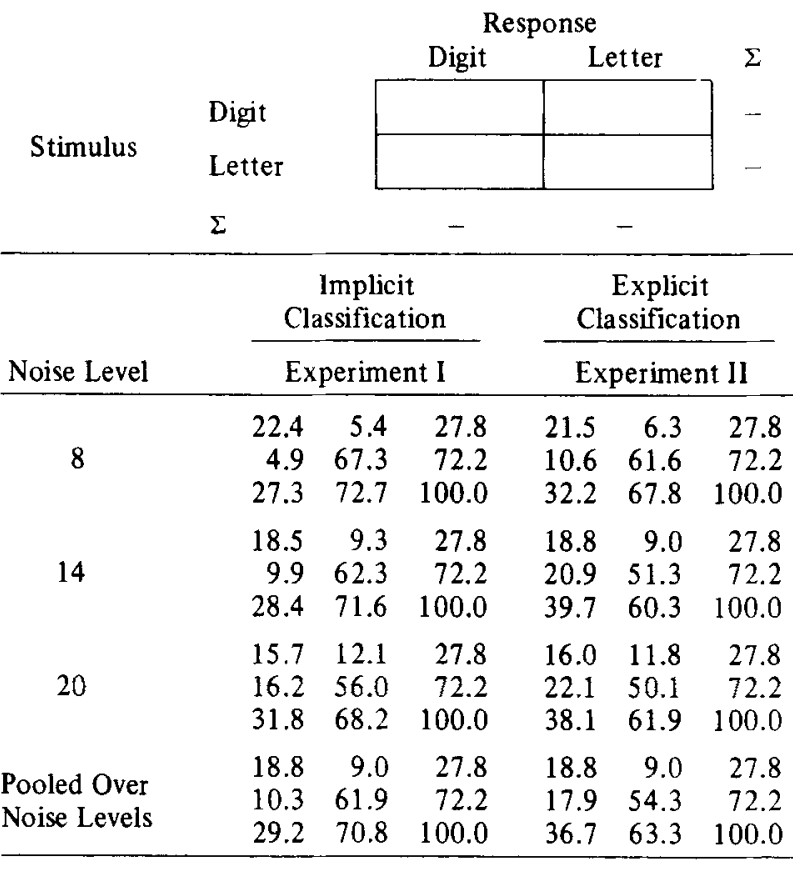

to the way that characters were constructed in this experiment. Certainly, characters comprised from dot patterns would not be as familiar to the Ss as those produced by standard type fonts. However, the characters were highly discriminable, and Ss had no difficulty in identifying them in the absence of visual noise. Moreover, the results are in keeping with those that were obtained by Dick (1971). The problem is to reconcile both of these results with findings such as those reported by Brand (1971) and Ingling (1972). In particular, how is it, if classification requires as much stimulus information as identification and if specifying a character's class requires more time than specifying its name, that visual search is more efficient when target and nontarget characters are in different classes than when they are in the same class?

One might account for Dick's finding in terms of the relative accessibility of the response codes. It may be that the name of a character is more accessible as a response to the visual representation of that character than is the name of the class to which the character belongs. This hypothesis attributes the RT difference that Dick obtained to a response encoding effect rather than to a stimulus processing effect. Response encoding time would have little importance in the visual scanning experiments of Brand and Ingling, inasmuch as the large majority of characters that were scanned did not require an overt response.

How to reconcile the present results with those of Brand and Ingling is less apparent. On the surface, they appear to be in conflict. Perhaps a key to the resolution 
is the fact that Ss were working under considerable time pressure in those experiments but not in the present one. In the latter case, Ss could examine the characters for a full second and could, therefore, take a more analytical approach to the task than was possible in Brand and Ingling's experiments. A study similar to the present one, but in which exposure duration is varied instead of noise level, might help to reconcile the findings.

\section{REFERENCES}

Atkinson, R. C., \& Shiffrin, R. M. Human memory: A proposed system and its control processes. In K. W. Spence and J. T. Spence (Eds.), The psychology of learning and motivation. Vol. II. New York: Academic Press, 1968.

Brand, J. Classification without identification in visual search. Quarterly Journal of Experimental Psychology, 1971, 23, 178-186.

Bruner, J. S., Goodnow, J. J., \& Austin, G. A. A study in thinking. New York: Wiley, 1956.

Dick, A. O. Relations between the sensory register and short-term storage in tachistoscopic recognition. Journal of Experimental Psychology, 1969, 82, 279-284.

Dick, A. O. Processing time for naming and categorization of letters and numbers. Perception \& Psychophysics, 1971, 9, 350-352.

Ingling, N. W. Categorization: A mechanism for rapid information processing. Journal of Experimental Psychology, 1972, 94, 239-243.

Posner, M. I. On the relationship between letter names and superordinate categories. Quarterly Journal of Experimental Psychology, 1970, 22, 279-287.

Snodgrass, J. G. Matching patterns vs matching digits: The effect of memory dependence and complexity on "same"-"different" reaction times. Perception \& Psychophysics, 1972, 11, 341-349.

Sperling, G., Budiansky, J., Spivac, J. G., \& Johnson, M. C. Extremely rapid visual search: The maximum rate of scanning letters for the presence of a numeral. Science, 1971, 174, 307-311.

(Received for publication February 11, 1973; revision received April 22, 1973.) 